\title{
Smart Workplaces for older adults: coping 'ethically' with technology pervasiveness
}

\author{
Sofia Segkouli ${ }^{1}$. Dimitrios Giakoumis ${ }^{1} \cdot$ Konstantinos Votis $^{1} \cdot$ Andreas Triantafyllidis $^{1} \cdot$ loannis Paliokas $^{1}$. \\ Dimitrios Tzovaras ${ }^{1}$
}

Accepted: 9 July 2021 / Published online: 20 July 2021

(c) The Author(s), under exclusive licence to Springer-Verlag GmbH Germany, part of Springer Nature 2021

\begin{abstract}
Pervasive technologies such as Artificial Intelligence, Virtual Reality and the Internet of Things, despite their great potential for improved workability and well-being of older workers, entail wide ethical concerns. Aligned with these considerations we emphasize the need to present from the viewpoint of ethics the risks of personalized ICT solutions that aim to remedy health and support the well-being of the ageing population at workplaces. The ethical boundaries of digital technologies are opaque. The main motivation is to cope with the uncertainties of workplaces' digitization and develop an ethics framework, termed SmartFrameWorK, for personalized health support through ICT tools at workplace environments. SmartFrameWorK is built upon a five-dimensional approach of ethics norms: autonomy, privacy, transparency, trustworthiness and accountability to incite trust in digital workplace technologies. A typology underpins these principles and guides the ethical decision-making process with regard to older worker particular needs, context, data type-related risks and digital tools' use throughout their lifecycle. Risk analysis of pervasive technology use and multimodal data collection, highlighted the imperative for ethically aware practices for older workers' activity and behaviour monitoring. The SmartFrameWorK methodology has been applied in a case study to provide evidence that personalized digital services could elicit trust in users through a well-defined framework. Ethics compliance is a dynamic process from participants' engagement to data management. Defining ethical determinants is pivotal towards building trust and reinforcing better workability and well-being in older workers.
\end{abstract}

Keywords Pervasive technology $\cdot$ Ethics framework $\cdot$ Workplaces $\cdot$ Older workers

Sofia Segkouli

sofia@iti.gr

Dimitrios Giakoumis

dgiakoum@iti.gr

Konstantinos Votis

kvotis@iti.gr

Andreas Triantafyllidis

atriand@iti.gr

Ioannis Paliokas

ipaliokas@iti.gr

Dimitrios Tzovaras

dimitrios.tzovaras@iti.gr

1 Centre for Research and Technology Hellas (CERTH), Information Technologies Institute-ITI, Thessaloniki, Greece

\section{Introduction}

An urgent need for digital tools to support older workers to remain active and healthy in their working environments is triggered by the demographic change in Europe and worldwide [1,2].

Statistics for the European Union indicate that the age structure population is expected to raise significantly over the next decades [3]. Thus, the EU has launched the notion of "Silver Economy" related to economic activities of people above 50 and their involvement on sectors of the national economy [4]. In this line, concerning ageing workforce productivity and workability enhancement, several Information and Communication Technology (ICT) systems are being proposed and developed to assess user specificities and needs in terms of ergonomics, health, safety and task assignments [5].

Beyond working efficiency, digital monitoring of employees' practices and performance may provide promising 
health and well-being outcomes [6]. Smart technologies such as Artificial Intelligence (AI), the Internet of Things (IoT), and Virtual Reality (VR) or Augmented Reality (AR) can strengthen the ageing workforce, significantly improving the ageing labour market and worker security [7]. According to Bejaković and Mrnjavac (2020) [8], digital skills, competences and literacy are critical in terms of seniors' successful participation in the digital society [9].

Despite the virtue of technologies on ageing workability sustenance and their physical and mental function, legal and ethical concerns are raised due to the sensitivity of working infrastructures and workers' rights protection. In terms of ICT solutions, these include large datasets collection, use and storage regarding the ownership and control of data due to potential data misuse. Within the sensitive area of health, self-tracking IoT technologies, as "computational sensing devices that track data about user behaviour to provide selfawareness' and support individuals and healthcare professionals, can negatively influence the person's privacy [10]. In addition, ethical risks are involved in technologies such as VR and AR [11], while addiction to digital services can infringe their stated purposes for promoting healthy behaviour [12].

These emerging ubiquitous and pervasive technologies (e.g. robotics, virtual/augmented reality, etc.) can exacerbate privacy issues or create novel ones, due to the increasing amount of data they create and collect while providing personalized services and support [13,14].

So far, relevant studies have focused on legal issues introduced by digital solutions [15]. Digital technologies' embodiment in the work environment has been strongly related to physical and psychosocial risks connected to the Occupational and Safety Health (OSH) field, e.g. through the continuous monitoring of workers via video cameras, apps and mobile devices. Although the intention of these technologies is to improve workers' safety, it could also allow invasion of privacy, which could also be psychologically detrimental. To this end, new methods and legal frameworks are required to address the pervasive technology-based new work forms [16], and deal with workplace surveillance.

Until now, the ethical dimensions of smart digital services for vulnerable social groups at their workplace, concern common ethics' values such as autonomy, beneficence, non-maleficence, justice [17]. In addition, the criticality of evaluating trade-offs in the workplace (e.g. increasing safety at the expense of privacy) has been stressed in relevant research. For instance, there is a trade-off between information loss and confidentiality, as reducing granularity results in diminished accuracy and utility of the data and its analysis [18]. Moreover, emphasis has been placed on risks emerging from pervasive computing-based infrastructures and services acknowledging the need for clear mitigation roadmaps [19]. Nevertheless, there is a gap in the examination of ethical challenges in workplaces for older adults in accordance with specific technologies. The necessity of such an examination is reinforced by the COVID-19 pandemic, during which several senior workers were required to work remotely, and an increased use of digital technologies was noticed [20].

Currently there is no ethical framework for ageing workers' workability and well-being monitoring through ICT tools. Given the criticality of personal data collection, ethical implications of these solutions should be addressed.

This article is structured as follows: In Sect. 2, we provide current knowledge about emerging pervasive ICT technologies ${ }^{1}$ involved at workplaces for worker performance and well-being monitoring. The main relevant ethical challenges are presented in Sect. 3, and ethical implications raised in the COVID-19 period are highlighted in Sect. 4. The outlined limitations and challenges prompted the introduction of a framework of ethics for older adults' monitoring through digital solutions, termed SmartFrameWorK, which is illustrated in Sect. 5. The applicability of the framework is highlighted in Sect. 6, through a case study presenting how this framework is built upon moral and transdisciplinary determinants, as well as the preferences, needs and digital capabilities of seniors, to define clear lines of data authorization and accessibility.

\section{Smart working environments and employees' monitoring through ICT technologies}

Pervasive Information and Communication Technologies (ICTs) can play a critical role in enabling power within workplaces. However, implications emerge from such technologies when used for worker performance appraisal systems [21]. The introduction of technology to organizational environments provided over years to managers and employees the opportunity to bring such demanding environments under control as studied by Orlikowski and Scott [22].

In particular, ICTs can provide new data collection and analysis tools in order to increase the visibility of employee behaviour and collect performance outcomes of employees [23].

\subsection{Personal loT devices for workers' monitoring}

Recent improvements in computing networking and the internet technologies have led the development of very powerful internet-connected devices, including sensors, smart phones, cameras and wearables. Wearable IoT devices can

\footnotetext{
$\overline{1}$ https://ec.europa.eu/info/sites/info/files/5._h2020_ethics_and_data_ protection.pdf.
} 
Table 1 Ethical concerns of digital technologies' use by senior silvers

\begin{tabular}{lll}
\hline & Concerns/ Risks & Measures to tackle with \\
\hline Personal IoT technologies & Privacy/ Invasion & Proper use/ Proportionality \\
& Trustworthiness/ Trust & Human agency \\
Ownership/ Control of data & Authorization, Transparency \\
& Freedom/ Empathy/ Disciplined & Relevance and suitability of data \\
Autonomy & Fairness/ Discrimination & $\begin{array}{l}\text { Accessibility to everyone } \\
\text { regardless to race, gender or } \\
\text { age }\end{array}$ \\
& $\begin{array}{l}\text { Sustainability of technologies } \\
\text { in the environment and the } \\
\text { Social stigma }\end{array}$ & society \\
\hline
\end{tabular}

be embedded as personal tools for instance to mining workers to provide proper protection from potential hazards [24]. Smart surveillance tools utilizing wearable devices and sensor technologies for tracking workers' data according to the health, location and environmental context of the worker have been used by several companies in the last years [25].

The use of wearable technologies at work has been introduced as a new form of employees' assessment allowing self-monitor and control abilities for workers' performance improvement and organizations' productivity aligned with workers' rhythms and needs [26].

Previous surveys have focused on the individual and organizational impact of the introduction of wearables. In particular, past works [27] have been dedicated mainly on improving workers safety through the surveillance of workers' physical parameters [28], stimulating physical activity, in terms of programs for wellness and quality of life [29] or detecting work-related stress and fatigue [30], while others aimed to improve ergonomics [31].

Relevant research investigated privacy issues caused by the monitoring capabilities of wearable technologies, as well as the implications of designing monitoring systems of workers [32]. As Marcengo and Rapp [33] pointed out, these concerns are most likely to be raised in a mass environment such as a workplace due to the fact that the technology for collecting, analysing and visualizing data is considered immature. In particular the collection of vast amount of data raises ethical questions such as how workers' data are used, who has access to the information and what are the consequences for the workers who do not meet the desirable indicators according to apps' interfaces. Therefore, issues of privacy, proper use (proportionality), minimization of data (relevant and suitable data), confidentiality, freedom and autonomy along with the scope of data analysis are highlighted as the main ethical challenges.

Using wearables as intervention tools to promote health can also bring significant implications for employees in terms of individual responsibility, self-belief, invasion of privacy and discrimination. To this end, the improvement of the design of workplaces and methods of collecting relevant data to inform such design is among the considerations of developers of diverse work domains. Concrete criteria have been defined to evaluate accessibility, adaptability and iterability of health wearable interfaces. From the perspective of ethics, this involves the cultural tendency of creating 'biotizens', namely gathering information about their body and then sharing that data with others to engage in 'proactive health self-management' [34].

Moreover wearables' use can be connected to socio-ethics challenges for the user. For instance, according to Mittelstadt [35], the social impact of using such devices for individuals deals with the stigma that this connection may in turn be associated with a health or disease condition.

Ethical concerns approached in a comprehensive manner are mainly related to personal IoT devices of older adults 'working environments' surveillance. These risks and the mitigation measures to cope with them, are presented in Table 1 according to the notion of the Sinek's 'Golden Circle' concept [36] and the five dimension approach of ethics.

Although the negative implications of wearable devices in the work environment gained much attention, the ethical dimensions of their use in the context of workplace systems are not clearly delineated. The ethical challenges of digital information transformation have been recently examined in relation to various moral dimensions such as the exposure of private life through access to data, the communication culture of algorithms, changes in the world of work [37]. However, systematic approaches on how ethical questions might influence the occupational progress of employees, and concrete measures/tools that have to be initiated as emerged from empirical evidence, have not been extensively researched [38].

\subsection{Augmented reality and virtual reality for workers' monitoring}

AR refers to the integration of the actual world with digital information, while VR refers to complete, 3-D virtual 
representations of the actual world or of objects within it [39]. The blending of physical with virtual worlds creates new opportunities for workplaces, towards improved ergonomics, training, collaboration and productivity. However, the use of innovative technological solutions such as AR and VR in workplaces has been primarily analysed from the perspective of workers' safety [40]. Only recently the ethical implications of the use of VR and AR technologies have been highlighted [41].

Furthermore, the technological evolution in fitness and well-being promotion brought the need for identifying potential ethical risks linked to virtual and augmented environments. Nevertheless, in previous examinations about virtual assistants' use the focus was mainly on theory-based approaches, not involving management considerations [42] about the pervasiveness of personalized assessment towards workability and Quality of Life (QoL). In addition, there is lack of tangible methods, practices and policies aligned with ageing workers' perceptions and against ageism to support ethical compliance and curation of sensitive data and metadata management, processing and governance.

\subsection{Artificial intelligence in surveillance at workplaces}

The availability of enormous computing power and vast amounts of data has made the integration of AI applications and tools in the workplace possible [43]. However, remote monitoring techniques that are based on AI, can be excessive if the context and the level of accountability have to be taken into consideration [44].

In particular, AI is among the primary technologies used in most business domains including daily services and decision support systems. Indisputably, ethical challenges emerge according to the design of these systems [45], their 'behaviour' as advanced autonomous systems [46], and the impact on decisions made without human involvement. Therefore, designers, engineers and researchers have to take into account these considerations when designing AI systems, in order to prevent the development of socially unacceptable systems with unpredictable behaviour. Ethical guidelines for AI ethical use along with safety mechanisms should be followed to avoid inappropriate use, control and limit systems' autonomy ${ }^{2}$ [47]

The role of AI researchers is key in the formulation of ethical machines, as ethics with regard to different specialties and sub-specialties need to be explored by developers and programmers [48]. In terms of this need, an ethical

\footnotetext{
${ }^{2}$ IEEE. (2016). Ethical Aligned Design. IEEE Standards Association. Available at: http://standards.ieee.org/develop/indconn/ec/ead_ v1.pdf.
}

framework supports the decision -making process [49]. More specifically, in the context of ICT for Active and Healthy Ageing (AHA), a number of ethical frameworks exploited the existing literature and policy documents as evidence of other projects. ${ }^{3}$

Nonetheless, current advances in AI call for giving specific attention to applied ethics. What is of high importance is to reinforce AI ethics with proper mechanisms in practice. Namely, ethics codes and principles have to prove their tangible impact when they are adapted and implemented in specific scenarios and/or research frame [50] Specifically in the era of an unexpected pandemic crisis, highly adaptive personalized ICT tools for workability and well-being improvement, have to be configured properly in order to be aligned with legal and ethical requirements, and secure workers' rights protection.

Digital monitoring of employees in the workplace needs to be aligned with transparency in the exclusive use of monitoring data to enhance workability. According to Lenca et al. [51], explicit acknowledgement of ethical values or considerations is largely absent in empirical research on monitoring technologies, and even when it is present, it is often confined to protecting human subjects in the immediate research encounter.

Therefore, the ethical implications of specific technologies have to be explored in order to indicate ethical choices that have to be made per technology case.

\section{Ethical dilemmas in specific technologies}

Among the high considerations of fundamental human rights are privacy, the right of individuals to control others' access to their personal information, and awareness through explicit consent given without coercion. Moreover, in the context of work, AI technologies that can optimize decision-making have to account ethical issues such as conflicts of interests and unintended consequences [52].

Another area of concern is AI involvement in tracking and predicting behaviour. AI can track and analyse data sources to inform decisions that could improve employees' productivity [53] Also excessive use of applications raises ethical issues to employees' monitoring according to voice recording, location tracking that can violate employees' privacy, while behaviour monitoring can increase stress on workers, create a climate of mistrust and pose risks to mental health [54].

Nonetheless, the demographic shift and the involvement of new technologies led to the need to revisit traditional legal

\footnotetext{
3 https://progressivestandards.org/wp-content/uploads/2018/10/Ethic al-Framework.pdf.
} 


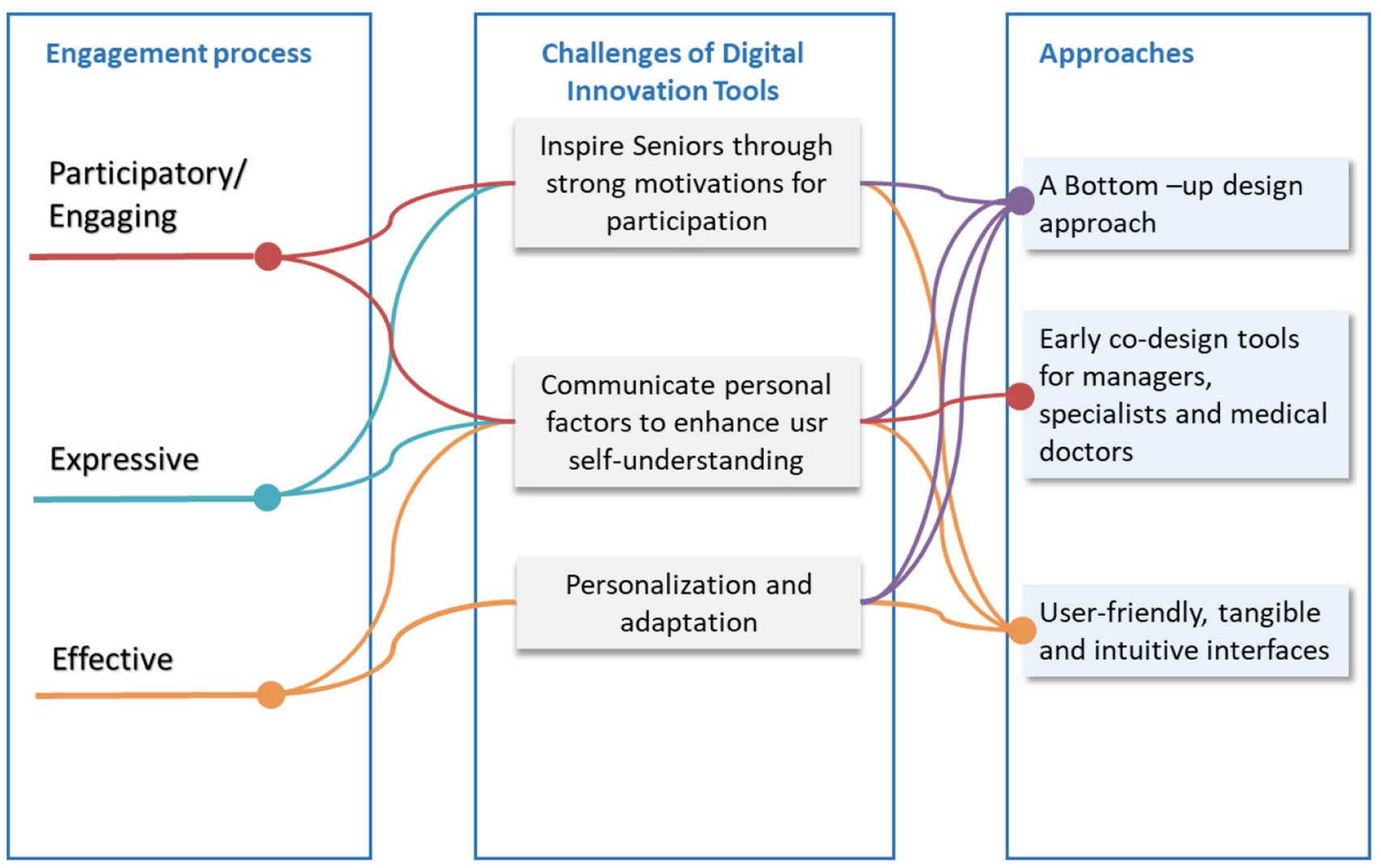

Fig. 1 Ethical challenges at workplace monitoring

and ethical principles related to occupational data and more specifically health data surveillance in liaison with interventions for health hazards' prevention.

As enabling technologies such as VR and AR are increasingly applied in the work environment to enhance workability and well-being, software developers, clinicians and researchers, should design and test carefully these tools upon a bottom -up design approach according to which activities are planned by seniors themselves ${ }^{4}$ The overall engagement process in order to be participatory should actively involve

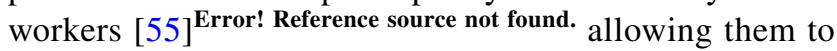
express their opinions on systems' design. This engagement process could result in greater "ownership" and commitment to any adaptations being implemented. The participatory process can be ethically based when it is well explained to the seniors from the start of the solution design, and as a result it can promote inspiration and motivation.

According to Article 29, in Opinion 8/2001, ${ }^{5}$ due to the nature of the relationship between employer and employee

\footnotetext{
${ }^{4}$ https://ec.europa.eu/health/ph_projects/2003/action1/docs/2003_1_ 26_frep_en.pdf.

5 https://ec.europa.eu/justice/article-29/documentation/opinionrecommendation/files/2001/wp48_en.pdf.
}

in case an employer has to process personal data of his/her employees it is misleading to start with the supposition that the processing can be legitimized only through the employees' consent. Even in cases where consent could be assumed to constitute a legitimate basis of an employee's data processing, the consent must be a clear and explicit expression of the employee's will. Default device settings and/or the installation of software enabling the electronic processing of employee's personal data under no circumstances can qualify as an employees' consent because consent requires an active expression of will. In general, the lack of action (i.e. no modification in default settings) should not be assumed to justify such processing.

Ethics on design of working performance visualization is of high importance, especially when it refers to the ageing population. Therefore, user interfaces have to be designed on an age-friendly notion to visualize individual features and factors in an intuitive and simple format (Fig. 1), for understanding respective personal specificities and providing a proactive and discreet behaviour towards active promotion of user physical and cognitive health, as the information of $\mathrm{OSH}$, managers, specialists and medical doctors.

Early co-design of digital coaching can inevitably support older adults. However, lack of acceptance, usability, and reliability of healthcare technologies remains an open issue 
and implies lack of respect in seniors' freedom and timing [56]. Furthermore, unexpected periods of global crisis, such as the one concerning the COVID-19 outbreak, which are associated with abrupt challenges, such as the practice of self-isolation, social distancing and remote working, demand the initiation of novel practices to promote the acceptance of technology [57].

\section{Ethical challenges in the COVID-19 era}

The pandemic crisis of COVID-19 forced one third of the world's population to live under 'lockdown' measures and was a major shock for the European and global economies. ${ }^{6}$ Over the world, industries faced significant financial loses [58], while societies were required to prioritize human resources and support vulnerable groups, such as seniors or people with chronic conditions, who were forced to work from home.

Home-based working as part of quarantine measures to reduce the potential exposure to COVID-19 [59], can have a tremendous effect on work-life balance [60]. In terms of quarantine measures older people have to remain vigilant which is also connected to determinant factors such as the nature of work performed and also to personality traits such as discipline and the competence to handle the assigned tasks successfully. Social isolation could be another deterrent factor to workability enhancement of ageing workers, which may feel loneliness and frustration away from their ordinary work culture [61].

Moreover, extended digitization of work procedures due to COVID-19 virus, brings new risks such as digital and social inequalities. Vulnerable populations, including older workers with chronic conditions, due to social isolation or poor socio-economic status, are more exposed to risks [62].

In terms of health emergencies and movement restrictions, remote monitoring, such as contact tracing, has been proposed as an effective practice, with ethical implications that have to be further analysed [63]. Intelligent monitoring of older adults' workability indicators could have a huge impact on work and social life landscape. However, ethical questions are raised by the extended use of digital solutions in daily life routine. An important concern has to do with individuals' privacy and freedom not only during the epidemic but also after it. Among the most important ethical challenges is to what extent privacy infringement will be justified under the scope of emergency and in which direction monitoring data, will be used and for how long.

\footnotetext{
$\overline{6}$ https://ec.europa.eu/info/business-economy-euro/doing-businesseu/jobs-and-economy-during-coronavirus-pandemic_en.
}

Considering the marginalization of senior silvers during the COVID-19 pandemic, due to psychological risks such as loneliness, isolation, ageism, and limitations to health care access, remote management of workability factors had to be enabled [64]. Ethical considerations in the COVID19 era and beyond demand new requirements for ethical frameworks that will be guided by specific contexts of locations, policies, resources and the extent of virus infection. For instance, business models of mild, moderate, severe and critical burden may be proposed. The same ethical principles that have always been used to guide transplant practices continue to apply during the COVID-19 era, but the balance between autonomy, beneficence, no maleficence, and justice depend on the policies, available resources, and local practices put in place. These principles provide a sound basis for a theoretical framework to guide and evaluate the digital well -being of older workers.

\section{The value of SmartFrameWork}

The significance of initiating a trustworthy framework of data processing applicable to pervasive technology systems is undeniable. In parallel, taking into account the complexity of workplace environments and the relevance of this complexity with the potential of technology pervasiveness, the need for a specific ethics framework emerged.

This theoretical framework has to be rigid according to ethics in a number of areas: (a) research engagement and participation; (b) data monitoring and tracking; (c) data processing during and after the study duration; (d) workplace relationships.

The convergence point was 'worker-older adult' needs and in order to unlock properly these needs, well-known theories have been explored such as the Bloom's Taxonomy about the Affective Domain to facilitate older workers' perceptions' understanding in depth as expressed at feelings, values, appreciation, enthusiasms, motivations, and attitudes.

These theoretical specificities shaped the 5-step design of the technologies of the study: (a) receiving with respect older adults' perception and doubts; (b) shaping the right and up to the point motivations; (c) demonstrating values and beliefs in a democratic process; (d) organizing values by resolving conflicts; (e) internalizing values by enabling teamwork, enhance commitment to ethical practice and its regular assessment.

After the initial five-step design of the study, the overall ethical implications and risks ranging from the engagement process to data governance and the final delivery of research in an ethical manner had to be addressed. Among the high considerations were the specific monitoring methods of workers' behaviour, workability and health status 


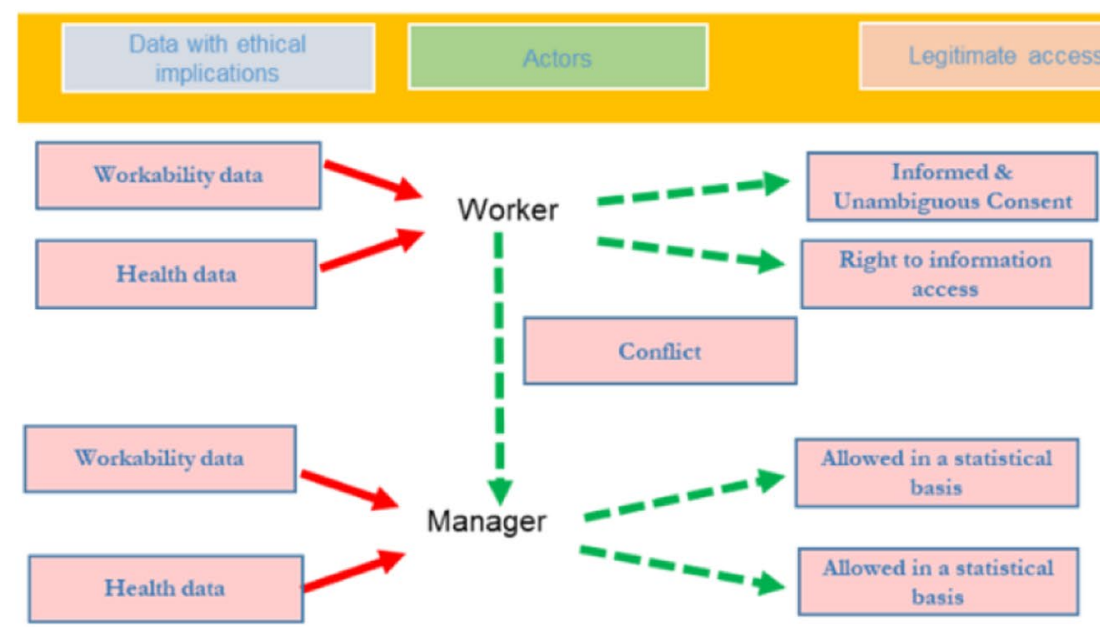

Fig. 2 Main pillars of the SmartFrameWork

through concrete technologies as well as the context of this monitoring. Furthermore, older workers' datasets were considered of high value and possibly sensitive activities' tracking, especially when applied in home environments, has to be defined.

Three pillars supported the theoretical framework: (a) datatype visibility, which entails ethical implications and are mostly prone to conflicts between workplace actors; (b) the actors who enable legitimate access to data and, (c) the scope of monitoring (Fig. 2).

According to processing operations through ICT usage, the blurred line between working life and activities outside the world has to become clear. Also the use in the workplace of office applications provided as a cloud service, which in theory allows for very detailed logging of the activities of employees along with the use of wearable devices (e.g. health and fitness devices) were protected by a number of measures that have been initiated and implemented on the core principles of 'limited access to sensitive data' and unobtrusiveness in AI technology involvement.

An ethical canvas has been structured to facilitate the process of potential ethical risks as indicated below (Fig. 3). Initially standard ethics' risks have been defined as emerged by the use of mixed emerging technologies along with the risks of ageist stereotypes, bias and discrimination that older adults experience and operate as obstacles in their wellbeing and health care support.

Furthermore, issues of data security and privacy are processed through a Secure Framework guided by two guidelines: (a) all personal and sensitive data will be stored to user's personal devices while (b) a carefull distinguishing is made among data derived from factory processes (e.g. machine operations, task assignments, etc.), and private user data.
Since data with ethical implications have been identified along with the ethical canvas of the data management at workplaces, there was also a provision for data fairness by making data accessible, interoperable, and reusable, including provisions for metadata analysis. In particular, the data generated through the worker activities monitoring platform during the whole lifecycle of the study are provisioned to be discoverable through a centralized database, part of the knowledgebase. Only accredited consortium members are permitted to have access to the data collections.

The main notion was to introduce an ethically-related framework for data management in the context of workplaces which will incorporate core user-centred principles

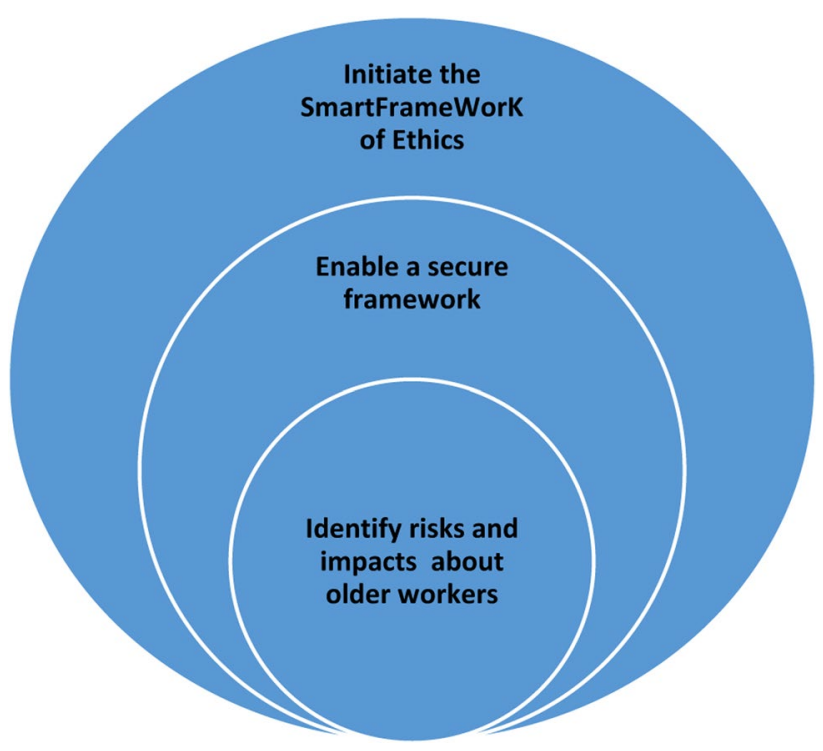

Fig. 3 The Ethical Canvas of data management at workplaces 
Table 2 A methodological approach for socio-ethical risks and opportunities' implementation on workplaces' environments

The 5 -step design implementation
a. Analysing with selected attention older adults' perception, doubts in terms of relevant surveys/question-
naires
b. Shaping the right and up to the point motivations taking into account the vulnerability of employees due
to their hierachical relationship to the employer within the specific workplace environment
c. Demonstrating values and beliefs in a democratic and diversity acceptance process through voluntary
participation and informed consent
d. Organizing values by resolving conflicts, exploring the importance of data protection when processing
sensitive personal data such as health data
e. Enhancing commitment to ethical practice and its regular assessment by setting up set-up ethical boards
and ethical assessment procedures to resolve ethical issues that likely would arise

such as ethical design awareness, human responsibility and accountability, data use upon decentralization, fairness and limited authorization and control by well-qualified researchers as well as support of ethical boards equipped with experts.

Moreover, data, metadata and documentation should follow disciplinary standards such as the open Web standards and the Common European Research Information Format (CERIF) metadata standard [65]. Data re-use is provisioned only through clarifying licenses while part of the anonymized data may be further analysed for statistical purposes while authorization and authentication have been defined in the frame of a decentralized data collection process. In particular, mobile apps should be used for interfacing the data collections of the IoT devices. Built upon the ethics by design principle, there is also provision that the storage of the monitoring data on the local mobile device (tablet or smartphone) and the right of sharing this data with the platform in order to receive the corresponding platform services (notifications, interventions, progress reports, awards etc.) should be up to each user responsibility.

\section{The SmartFrameWorK use case}

The ethical framework designed to complement secure data management of older workers has been applied in the use case of the EU-funded project, Ageing@Work [5], which is based on the urgent need to support ageing workers of the modern industries to maintain and retain productivity and workability. To this end, novel advanced, personalized ICT solutions for adaptive smart working and living environments supporting active and healthy ageing are being deployed according to workers' needs.

The main objective herein is to analyse ethical implications related to integrated platforms of advanced, personalized and adaptive ICT tools, which aim to improve the wellbeing and productivity on the basis of AI, AR/VR, and IoT wearable devices, and tailor the workplace to the evolving needs and specificities of the ageing workers.
Large companies as representatives of the industrial area and part of the present study support two diverse professional cases, the office ergonomics and the use case of mining to gain insights regarding the different organization ethics challenges and their impact on employers-employees relationships and human resource areas. In the light of improved productivity and process efficiency attention has to be paid on the design of an ethical approach and methodology regarding data and metadata management of older workers.

Therefore, the SmartFrameWork has to be implemented taking into account the socio-ethical risks, current unexpected societal crises such as COVID-19 and its reflections to human resource management at workplaces.

For this reason, an initial analysis of topics concerning ethics and data protection in relation to each professional case and its particular ethical aspects has been conducted in accordance with the 5-step design as depicted in Table 2.

Due to the increased exposure in distant monitoring of workability, a need for constant evaluation of data use awareness emerged towards personal security preservation. Thus, the methodological approach has to be adjusted placing emphasis on the critical role of ethics experts. Older workers' willingness and self-imposed engagement have to be supported through meaningful information, communicating in a fair way the trade-offs of services offered under these new circumstances.

Beyond the regulatory process and the complemented instruments, a conceptual procedure has to be followed in order to frame the ethical values/standards of older workers activity and behaviour-monitoring system design and its use. Crucial activities have been established in terms of data management such as cultivating and implementing an ethical culture with ethical experts and Ethical Boards' consultation and support, defining and applying ethical values in the daily research practice to ensure the appropriateness of decisions taken for data management of older adults' workers. The outcome of this process was a specific framework of ethics for seniors monitored at workplaces and homes (Fig. 4). 


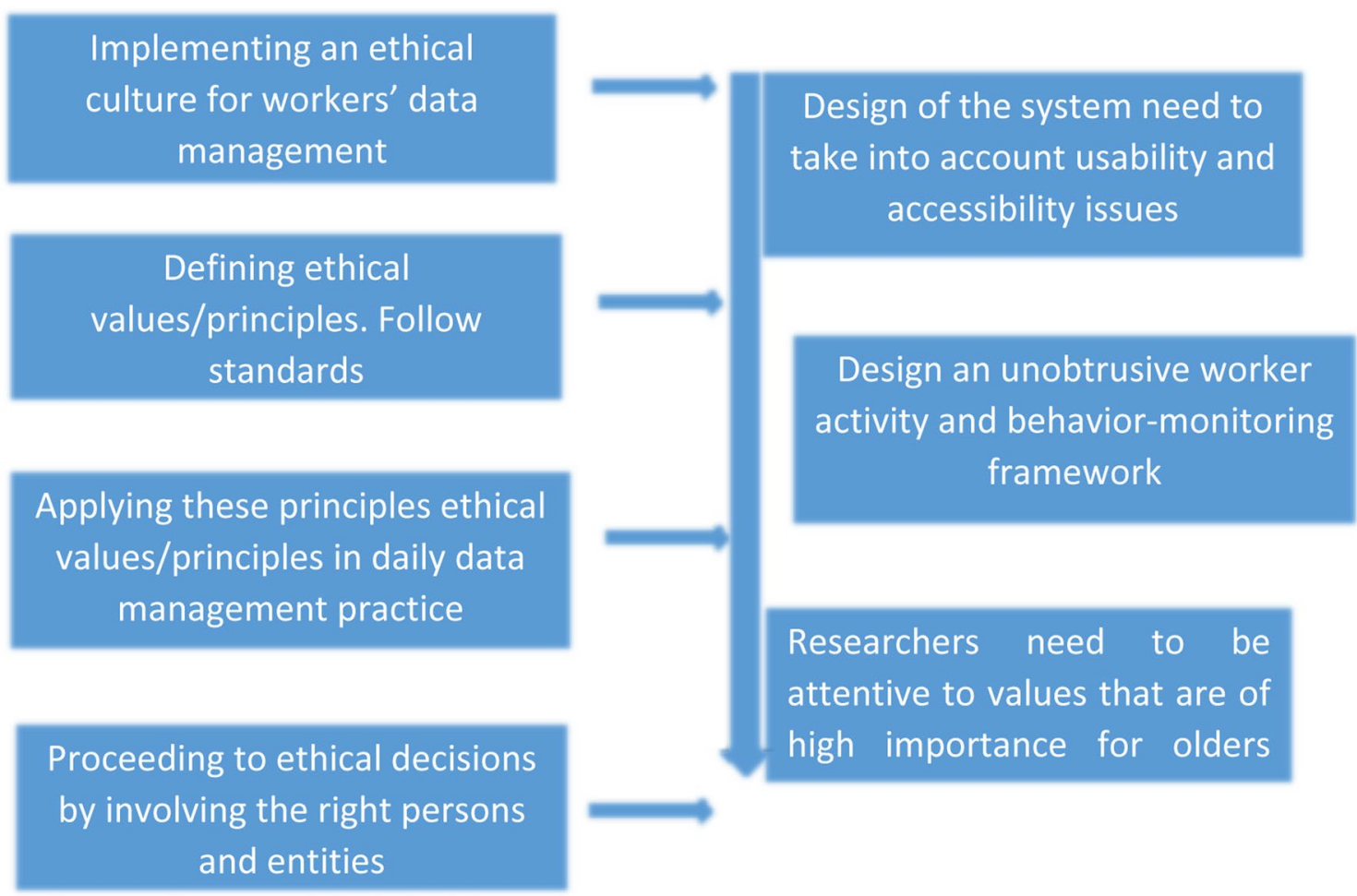

Fig. 4 An ethical framework of remote monitoring of older workers

In addition to workers' satisfaction, ethics have been approached in liaison with system specification and architecture, ethics by design and data management upon human/ workers' rights' preservation. The main aim was to initiate self-tracking devices that can be less threatening, in ethical terms, emphasizing on user engagement upon a voluntary basis and providing a high level of control when sensitive information is monitored and tracked. Moreover, emphasis is put on the potential role that specific organizational influences and strategic plans can play to advance, secure and explore the ethical imperatives for protecting privacy as well as well-being issues of seniors.

Taking into account that potentially confidential information according to the workplace design and work processes will be recorded and processed, an urgent need emerges for protecting strict confidentiality and participants' dignity preservation. Relevant manuals of ethics along with ethics protocols of the study have been provisioned from the beginning of this study in the line of core values as Ethics by design and Ethics by default. However, apart from these minimum requirements, and the alignment with international guidelines and codes of conduct such as Ethical Guidelines for Health-related Research Involving Humans (World
Health Organization $)^{7}$ and Standards and Operational Guidance for Ethics Review of Health-Related Research with Human Participants [66], more demanding issues emerged according to data processing of ageing workers' workability through adaptive personalized ICT tools.

In the context of business ethics as a form of applied ethics the three-dimensional problem of ethics: (a) micro (personal) issues, (b) meso (organizational) and, finally (c) the long-term impact of ethics [67] from a macro perspective side (sector-wide/society) has been integrated in the methodology of workability data management.

According to normative literature the use case study aimed at the initiation and implementation of appropriate ethical strategies to establish the Ethical Framework as a guide for data monitoring, control and validation in line with vigilant compliance with ethics and law requirements. The main objectives were to establish basic ethics and privacy guidelines aligned with the original purpose of the use case study.

Privacy and confidentiality in the workplace were among the critical concerns for both employers and employees. Therefore, in the light of smart working environments

\footnotetext{
7 World Health Organization, \& Council for International Organizations of Medical Sciences. (2016). International ethical guidelines for health-related research involving humans.
} 


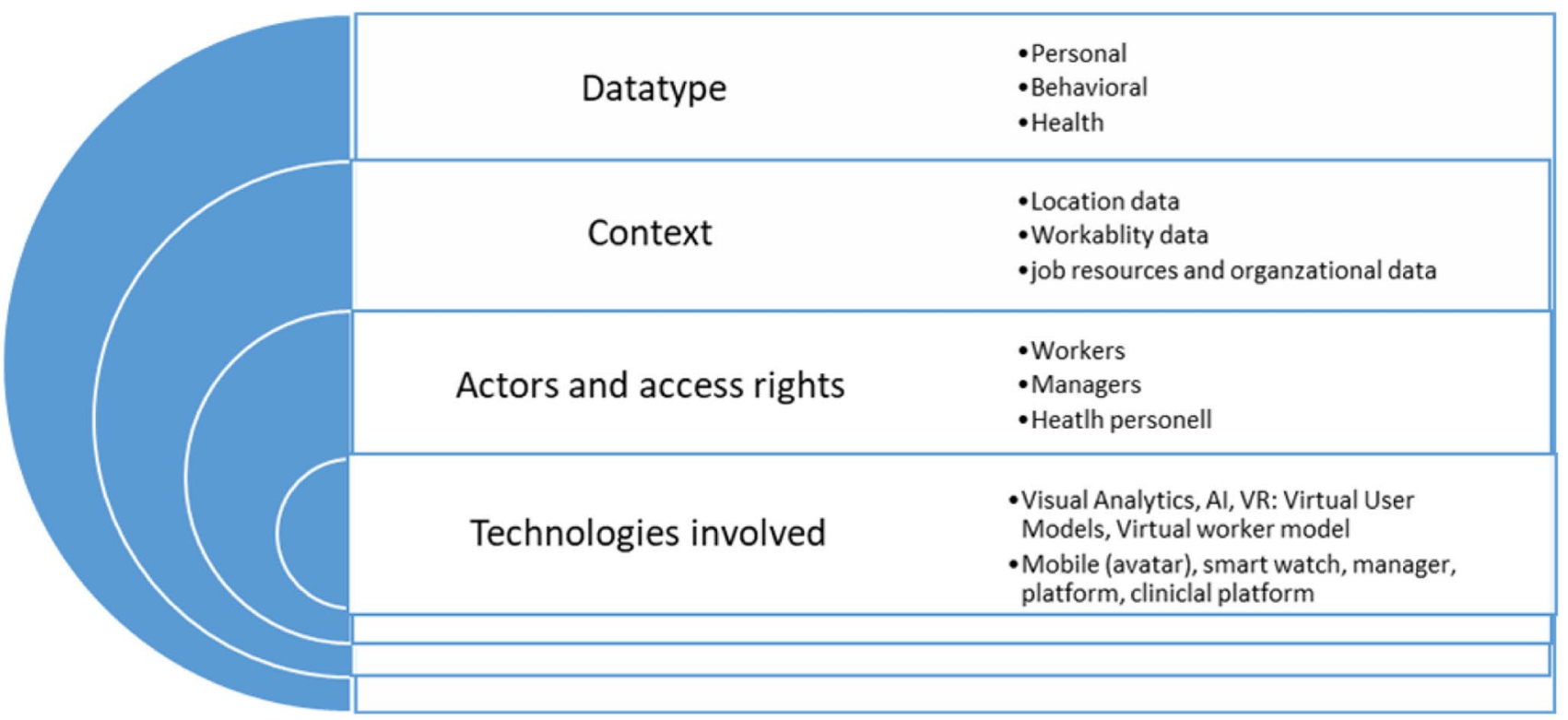

Fig. 5 Typology of multimodal datasets according to datatype and the context of data collection

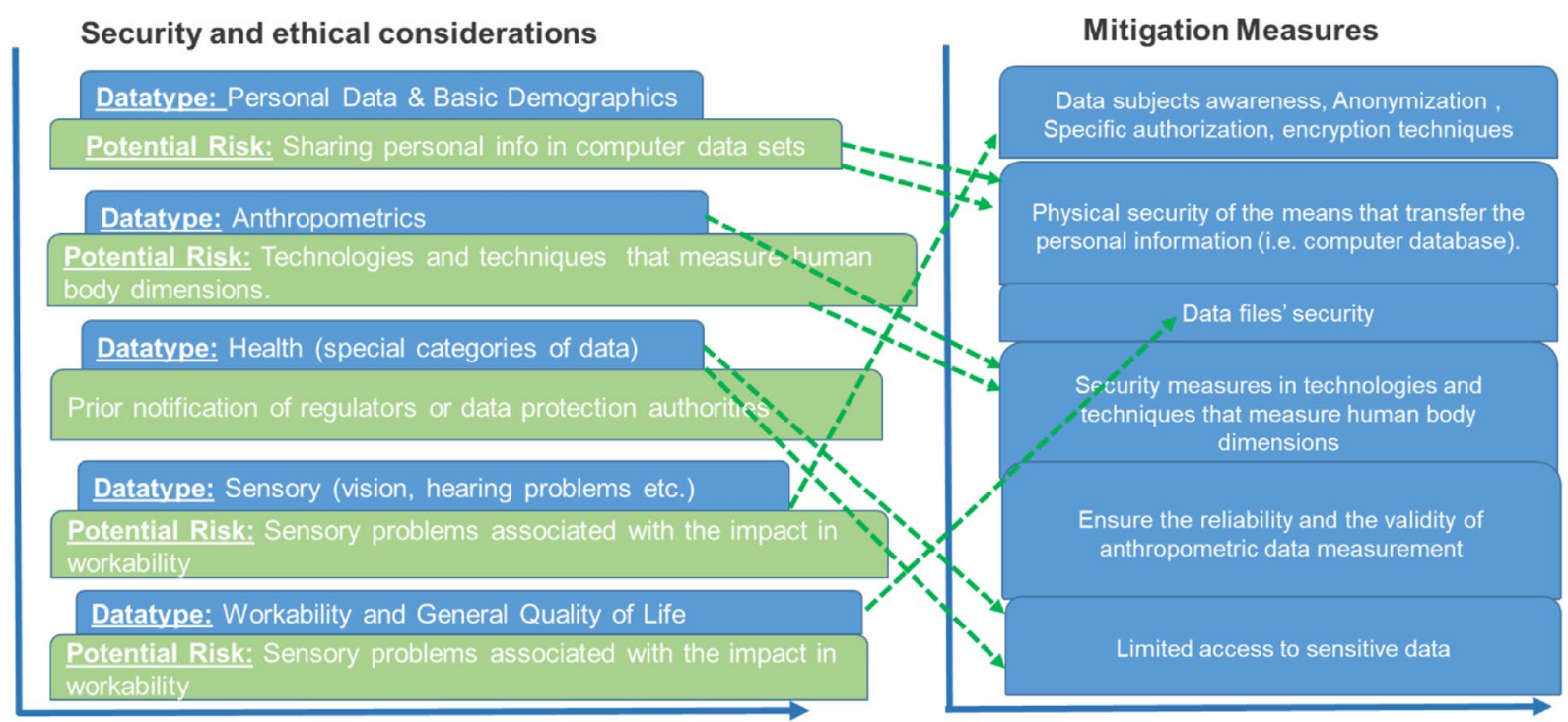

Fig. 6 Identification of datatypes, ethical Implications and Measures in Smart working environments by the use case study

through ICT technologies' use, a typology has been specified according to a number of categorical variables, including (a) data type, (b) context of data, (c) specific technologies and, (d) actors involved in the collection and processing of this data (Fig. 5).

The main ethical considerations of data management were interrelated with security issues, i.e. privacy, data protection and safety. Therefore, a clear definition of each security and ethical concern in accordance with the proper mitigation measures have been outlined as presented in Fig. 6 .
Overall, data convey the stress level and the average productivity levels while type of data and data processing purposes out of the scope of GDPR, such as data for statistical purposes, have been defined from the start. Particular attention has been paid to special categories of data processing (formerly known as 'sensitive data'), profiling, automated decision-making, data-mining techniques, bigdata analytics and artificial intelligence, as such processing may entail higher risks to fundamental human rights. Moreover dealing with and respecting users' privacy for 
affective data raised concerns for stress related to digital technologies' use [68].

A thorough mapping of all data along with the technologies involved was valuable in understanding the potential risks of data use and the limitations that had to be defined according to social sensitive groups as older adults and the access to their data. These specific parameters according to workers' datasets use that may implicate ethical concerns are quoted below:

- Involvement of technologies and techniques that measure human body dimensions;

- Verification of reliability and validity of anthropometric data measurement;

- Sensory problems processing that might be associated with the impact in workability;

- Lifestyle habits' monitoring as part of public health monitoring;

- Monitoring and measuring occupational health data;

- Measuring occupational health and safety risks.

Data-related risks have been considered in accordance with each phase of the data cycle from the collection, processing, storage, to the analysis and use of data in terms of risks assessment and decision-making. In the current study, specific challenges were faced according to two clusters of information, (a) overall ethics practices including unclear 'grey areas' of AI and VR technologies use and (b) information access, in particular manager rights to this access through the core technologies (i.e. apps' interfaces, ageing workers virtual user models, etc.).

Design principles and guidelines such as respect to privacy rules had to be followed by the designers and developers in order to ensure balance between personalization and privacy. In particular, personalized recommendations concerning for example physical activity and breaks at the workplace were provided by a virtual coach (avatar) to workers in a mobile app based on well-structured data and clearly-defined rules evaluated through co-designed surveys. Concerning the multimodal data received through the mobile app, a privacy policy regulated the terms of app use providing concrete information about datatype collected by the mobile app, the methods and duration of data collection and also location information such as latitude, longitude or addresses. According to this risk, limitations were defined so that raw sensitive information was never shared with the server, but instead stored locally in worker's phone. Furthermore, data provided by third party applications such as Google (e.g. for activity recognition) was permitted only with user explicit consent. Moreover, anonymity along with authentication and authorization mechanisms was applied in order to avoid user identification, thereby reinforcing the trust of the users on the system. An indicative scenario illustrating the recommendations for breaks at the workplace provided by our system was published as a YouTube video. ${ }^{8}$

\section{Concluding remarks}

Smart workplaces employing pervasive technologies raise a number of ethical issues, which should be viewed more systematically. The present paper, in response, provides a holistic point of view about the research conduct ad hoc, ranging from older adults' active engagement in a participatory monitoring system, to the preparedness of researchers to store, manage, and analyse personal data.

The use of digital solutions enabled by web, smart mobile devices, wearable sensors and AI technologies, needs to meet ethical requirements according to individuals' engagement in the monitoring process, such as the protection of autonomy, freedom, dignity of seniors and initiate proper safeguards to data governance and management of working performance inside and outside the working environment. To achieve this social value, ageing workers' fair and accurate awareness about the risks and benefits of digital technologies' use is a prerequisite.

Our particular focus was on ethics of digitized workplaces for older adults. Ethical aspects have been identified in accordance with smart, personalized and adaptive ICT solutions for active, healthy and productive ageing, and an ethical framework has been defined and developed as the basis of effective digital workplaces' strategies in alignment with wider human values such as sense of control, trust, sense of freedom and ownership which converge with older workers meaningfulness of work and success. More systematic approaches have been proposed through a specific theoretical framework designed in accordance with ethical culture and ethical behaviour towards enabling fair, human-centred data management, and promoting ethical assessment which can bring balance between work and personal life.

In conclusion, this paper introduced an ethics digital-specific framework for older workers, to support the benefits of pervasive monitoring in their well-being and productivity, within our ageing societies. The framework encountered core principles such as privacy, autonomy and data proportionality to frame older workers' privacy rights at working environments. Built upon moral and transdisciplinary determinants as well as the preferences, needs and digital capabilities of seniors, the developed framework aims to contribute in the design of ethically-aware digital practices, policies, and tools for workplaces.

\footnotetext{
${ }^{8}$ https://www.youtube.com/watch?v=T4uh4nuII70
} 
Acknowledgements This paper is part of the Ageing@Work, Smart, Personalized and Adaptive ICT Solutions for Active, Healthy and Productive Ageing with enhanced Workability, RIA project that has received funding from $\mathrm{H} 2020-\mathrm{SC} 1-\mathrm{DTH}-2018-1$.

Funding This work has been supported by the EU Horizon 2020 funded project "Smart, Personalized and Adaptive ICT Solutions for Active, Healthy and Productive Ageing with enhanced Workability (Ageing@Work)”, under Grant Agreement No. 826299.

\section{Declaration}

Conflict of interest On behalf of all authors, the corresponding author states that there is no conflict of interest.

\section{References}

1. Dantas, C., van Staalduinen, W., Machado, N., Jegundo, A., \& Rodrigues, F. (2019). Older workers, technology and the balance of power: an ethical review. In: Proceedings of the 12th ACM International Conference on PErvasive Technologies Related to Assistive Environments (pp. 464-467).

2. Dundon, T., Martinez Lucio, M., Howcroft, D., Hughes, E., Keizer, A., \& Walden, R. (2017). Power dynamics in work and employment relationships: The capacity for employee influence.

3. ULTAN, M. Ö. Demographic change analysis of the European union and future projections.

4. Bojanić, I. B., \& Erceg, A. (2017). Silver economy: Demographic change and economic opportunity. Under the auspices of the President of the Republic of Croatia, 1005.

5. Giakoumis, D., Votis, K., Altsitsiadis, E., Segkouli, S., Paliokas, I., \& Tzovaras, D. (2019, June). Smart, personalized and adaptive ICT solutions for active, healthy and productive ageing with enhanced workability. In: Proceedings of the 12th ACM International Conference on PErvasive Technologies Related to Assistive Environments (pp. 442-447).

6. Queiros, A., Dias, A., Silva, A.G., Rocha, N.P.: Ambient assisted living and health-related outcomes-a systematic literature review. Informatics 4(3), 19 (2017). https://doi.org/10.3390/infor matics 4030019

7. Rinaldi, A., \& Kianfar, K. (2020, July). Design of digital coaches for health and wellness in the workplace. In: International Conference on Human-Computer Interaction (pp. 135-146). Springer, Cham.

8. Bejaković, P., \& Mrnjavac, Ž. (2020). The importance of digital literacy on the labour market. Employee Relations: The International Journal.

9. Chawla, N.V., Davis, D.A.: Bringing big data to personalized healthcare: a patient-centered framework. J. Gen. Intern. Med. 28(3), 660-665 (2013)

10. Mortenson, W.B., Sixsmith, A., Woolrych, R.: The power (s) of observation: Theoretical perspectives on surveillance technologies and older people. Ageing Soc. 35(3), 512-530 (2015)

11. Morley, J., Joshi, I.: Developing effective policy to support artificial intelligence in health and care. Eurohealth 25(2), 11-14 (2019)

12. Oravec, J.A.: Digital iatrogenesis and workplace marginalization: some ethical issues involving self-tracking medical technologies. Info. Commun. Soc. 23(14), 2030-2046 (2020). https://doi.org/ 10.1080/1369118X.2020.1718178

13. Burr, C., Taddeo, M., Floridi, L.: The ethics of digital well-being: A thematic review. Sci. Eng. Ethics 26(4), 2313-2343 (2020). https://doi.org/10.1007/s11948-020-00175-8
14. Stahl, B.C., Timmermans, J., Flick, C.: Ethics of emerging information and communication technologieson the implementation of responsible research and innovation. Sci. Public Policy 44(3), 369-381 (2017)

15. Royakkers, L., Timmer, J., Kool, L., van Est, R.: Societal and ethical issues of digitization. Ethics Inf. Technol. 20(2), 127-142 (2018)

16. Mattila-Wiro, P., Samant, Y., Husberg, W., Falk, M., Knudsen, A., \& Saemundsson, E. (2020). Work today and in the future: perspectives on occupational safety and health challenges and opportunities for the nordic labour inspectorates.

17. Jones, S., Hara, S., Augusto, J.C.: eFRIEND: an ethical framework for intelligent environments development. Ethics Inf. Technol. 17(1), 11-25 (2015)

18. Gostin, L.O., Levit, L.A., Nass, S.J. (eds.): Beyond the HIPAA privacy rule: enhancing privacy, improving health through research. National Academies Press (2009)

19. Ortet, S., Dantas, C., Machado, N., Tageo, V., Quintas, J., \& Haansen, S. (2019, June). Pervasive technologies applied to the work environment: Implications for end-users: the foreground for SmartWork concerns and requirements. In: Proceedings of the 12th ACM International Conference on PErvasive Technologies Related to Assistive Environments (pp. 459-463).

20. Budd, J., Miller, B.S., Manning, E.M., Lampos, V., Zhuang, M., Edelstein, M., Short, M.J.: Digital technologies in the publichealth response to COVID-19. Nat. Med. 26(8), 1183-1192 (2020)

21. Addabbo, T., Ales, E., Curzi, Y., Fabbri, T., Rymkevich, O., Senatori, I.: Performance Appraisal in Modern Employment Relations. Springer International Publishing (2020)

22. Orlikowski, W.J., Scott, S.V.: Sociomateriality: challenging the separation of technology, work and organization. Acad. Manag. Ann. 2, 433-474 (2008)

23. Miele, F., Tirabeni, L.: Digital technologies and power dynamics in the organization: A conceptual review of remote working and wearable technologies at work. Sociol. Compass 14(6), e12795 (2020)

24. Georgakopoulos, D., Jayaraman, P.P.: Internet of things: from internet scale sensing to smart services. Computing 98(10), 1041-1058 (2016)

25. Roberto, A., Curzi, Y., Parisi, T., Tirabeni, L.: Perceived autonomy and discretion of mobile workers. Studi Organizzativi 2, 31-61 (2018)

26. Lupton, D. (2014). Self-tracking cultures: towards a sociology of personal informatics. In: Proceedings of the 26th Australian computer-human interaction conference on designing futures: The future of design (pp. 77-86).

27. Gundars, K., Coco, M.: Perceptions of internetof-tings surveillance by human resource managers. SAM Adv. Manag. J. 82, 53-64 (2017)

28. Wilson, H. James (2013). "Wearables in the Workplace." Harvard Business Review, September.

29. Laurie G., Gonzalez E., and Leidner D. (2016). "I Track, Terefore I Am: Exploring the Impact of Wearable Fitness Devices on Employee Identity and Well-Being." 22th Americas Conference on Information Systems, San Diego, August.

30. Lu, H., Zhang, Q., Chen, X., et al.: Detecting work-related stress with a wearable device. Comput. Ind. 90, 42-49 (2017)

31. Peppoloni, L., Filippeschi, A., Rufaldi, E., Avizzano, C.A.: A novel wearable system for the online assessment of risk for biomechanical load in repetitive eforts. Int. J. Ind. Ergon. 52, 1-11 (2016)

32. Kotler, P., \& Armstrong, G. (2010). Principles of marketing. Pearson education.

33. Marcengo, A., Rapp, A.: Visualization of human behaviour data. Int. J. Commun. 8, 236-265 (2014) 
34. Lupton, D.: Digitized health promotion: personal responsibility for health in the web 2.0 era. Sydney Health \& Society Group (2013)

35. Mittelstadt, B.: Ethics of the health-related internet of things: a narrative review. Ethics Inf. Technol. 19(3), 157-175 (2017)

36. Straker, K., Nusem, E.: Designing value propositions: An exploration and extension of Sinek's 'Golden Circle'model. J. Design. Business. Soc. 5(1), 59-76 (2019)

37. Bedford-Strohm, H.: The ethical challenges of the digital age: between promises of salvation and prophecies of doom. Ecum. Rev. 72(2), 167-182 (2020)

38. Nikayin, F., Heikkilä, M., De Reuver, M., Solaimani, S.: Workplace primary prevention programmes enabled by information and communication technology. Technol. Forecast. Soc. Chang. 89, 326-332 (2014)

39. Farshid, M., Paschen, J., Eriksson, T., Kietzmann, J.: Go boldly!: Explore augmented reality (AR), virtual reality (VR), and mixed reality (MR) for business. Bus. Horiz. 61(5), 657-663 (2018)

40. Lanyi, C.S., Withers, J.D.A.: Striving for a safer and more ergonomic workplace: acceptability and human factors related to the adoption of AR/VR glasses in industry 4. 0. Smart Cities 3(2), 289-307 (2020). https://doi.org/10.3390/smartcities3020016

41. Carter, M., \& Egliston, B. (2020). Ethical implications of emerging mixed reality technologies.

42. Papa, A., Mital, M., Pisano, P., Del Giudice, M.: E-health and wellbeing monitoring using smart healthcare devices: An empirical investigation. Technol. Forecast. Soc Change 153, 119226 (2020)

43. Kim, P.T.: Big Data and artificial intelligence: New challenges for workplace equality. U. Louisville L. Rev. 57, 313 (2018)

44. Vatcha, A. Workplace Surveillance Outside the Workplace: An Analysis of E-Monitoring Remote Employees. EDITORIALFrom the Faculty Editor, 4.

45. Bostrom, N., and Yudkowsky, E. (2014). "The ethics of artificial intelligence," in The Cambridge Handbook of Artificial Intelligence, eds F. Keith and M. William (Ramsey: Cambridge University Press), 2014.

46. Wallach, W., Allen, C.: Moral Machines: Teaching Robots Right from Wrong. Oxford University Press (2009)

47. Bryson, J., Winfield, A.F.: Standardizing ethical design for artificial intelligence and autonomous systems. Computer 50, 116-119 (2017). https://doi.org/10.1109/MC.2017.154

48. Anderson, M., Anderson, S.L.: Machine Ethics. Cambridge University Press (2011)

49. Grill, K., Dawson, A.: Ethical frameworks in public health decision-making: defending a value-based and pluralist approach. Health Care Anal. 25(4), 291-307 (2017)

50. Hagendorff, T.: The ethics of Ai ethics: An evaluation of guidelines. Minds. Mach. 30(1), 99-120 (2020). https://doi.org/10. 1007/s11023-020-09517-8

51. Lenca, M., Wangmo, T., Jotterand, F., Kressig, R.W., Elger, B.: Ethical design of intelligent assistive technologies for dementia: a descriptive review. Sci. Eng. Ethics 24(4), 1035-1055 (2018)

52. Christoph, J. N. E. Ethics and Workplace Automation.

53. Metcalf, L., Askay, D.A., Rosenberg, L.B.: Keeping humans in the loop: pooling knowledge through artificial swarm intelligence to improve business decision making. Calif. Manage. Rev. 61(4), 84-109 (2019)

54. Booth, R.: UK businesses using artificial intelligence to monitor staff activity. The Guardian 7, 2019 (2019)
55. Burgess-Limerick, R.: Participatory ergonomics: evidence and implementation lessons. Appl. Ergon. 68, 289-293 (2018)

56. Santini, S., Galassi, F., Kropf, J., Stara, V.: A digital coach promoting healthy aging among older adults in transition to retirement: results from a qualitative study in Italy. Sustainability 12(18), 7400 (2020)

57. Anka, A., Thacker, H., Penhale, B.: Safeguarding adults practice and remote working in the COVID-19 era: challenges and opportunities. J. Adult Protect. 22(6), 415-427 (2020). https://doi.org/ 10.1108/JAP-08-2020-0040

58. Wuest, T., Kusiak, A., Dai, T., \& Tayur, S. R. (2020). Impact of COVID-19 on Manufacturing and Supply Networks-The Case for AI-Inspired Digital Transformation. Available at SSRN 3593540.

59. Monahan, C., Macdonald, J., Lytle, A., Apriceno, M., Levy, S.R.: COVID-19 and ageism: How positive and negative responses impact older adults and society. Am. Psychol. 75(7), 887-896 (2020). https://doi.org/10.1037/amp0000699

60. Palumbo, R., Manna, R., Cavallone, M.: Beware of side effects on quality! Investigating the implications of home working on work-life balance in educational services. TQM J. 33(4), 915-929 (2020). https://doi.org/10.1108/TQM-05-2020-0120

61. Barakat, D. E. (2014). Telecommuting ethics and work-family conflicts-family-work conflicts. Theses, Dissertations, and Projects.

62. Robinson, L., Schulz, J., Khilnani, A., Ono, H., Cotten, S.R., Mcclain, N., Tubaro, P.: Digital inequalities in time of pandemic: COVID-19 exposure risk profiles and new forms of vulnerability. F. M. (2020). https://doi.org/10.5210/fm.v25i7.10845

63. Parker, M.J., Fraser, C., Abeler-Dörner, L., Bonsall, D.: Ethics of instantaneous contact tracing using mobile phone apps in the control of the COVID-19 pandemic. J. Medical. Ethics. 46(7), 427-431 (2020). https://doi.org/10.1136/medethics-2020-106314

64. Panico, F., Cordasco, G., Vogel, C., Trojano, L., Esposito, A.: Ethical issues in assistive ambient living technologies for ageing well. Multimed. Tools. Appl. 79(47-48), 36077-36089 (2020). https://doi.org/10.1007/s11042-020-09313-7

65. Jörg, B.: CERIF: The common European research information format model. Data. Sci. J. 9, 24-31 (2010). https://doi.org/10. 2481/dsj.CRIS4

66. Lynch, H.F., Abdirisak, M., Bogia, M., Clapp, J.: Evaluating the quality of research ethics review and oversight: a systematic analysis of quality assessment instruments. AJOB. Empirical. Bioethics. 11(4), 208-222 (2020)

67. Appelbaum, S.H., Vigneault, L., Walker, E., Shapiro, B.T.: (Good) corporate governance and the strategic integration of meso ethics. Soc. Responsib. J. 5(4), 525-539 (2009). https://doi.org/10.1108/ 17471110910995366

68. Poguntke, R. (2020). Understanding stress responses related to digital technologies.

Publisher's Note Springer Nature remains neutral with regard to jurisdictional claims in published maps and institutional affiliations. 